\title{
Acute pancreatitis-presentation as a discoloured lump in the groin
}

\author{
ASHLEY R. DENNISON \\ F.R.C.S.
}

GAVIN T. ROYLE

M.S., F.R.C.S.

Nuffield Department of Surgery, University of Oxford, John Radcliffe Hospital, Headington, Oxford OX3 9DU

\section{Summary}

Pancreatitis presenting as a mass in the inguinal region is extremely rare. Such a case in an adult male is presented and the diagnostic problems discussed.

KEY WORDS: alcohol, inguinal canal.

\section{Introduction}

The clinical presentation of acute pancreatitis is very variable, and the diagnosis can be difficult to make (Joshi, Probstein and Blumenthal, 1957). Physical signs that may present in the abdomen include those of skin discoloration in the flanks (Grey Turner, 1919-20) and around the umbilicus (Cullen, 1919).

We report a case where the presentation was that of a tender discoloured lump in the groin.

\section{Case report}

A male engineer aged 54 years was admitted as an emergency because of 10 days abdominal pain, a tender lump in the left groin for 7 days, and vomiting since a few hours after the appearance of the lump.

He was well before this illness although for 3-4 months he had been prescribed an antacid for some intermittent 'gastritis'. He was married, did not smoke and described his alcohol intake as minimal.

On examination on admission he appeared extremely unwell. He was cyanosed, dehydrated, pyrexial at $37.8^{\circ} \mathrm{C}$, shocked with a blood pressure of $90 / 60$ $\mathrm{mmHg}$, and had a weak radial pulse of 110 per min.

His abdomen was distended, with a $5 \mathrm{~cm}$ sausage shaped lump in the region of the left inguinal canal. The overlying skin was red, there was no cough impulse and the lump was irreducible. Bowel sounds were absent and his abdomen was diffusely tender to deep palpation.

Investigations showed a plasma amylase of less than 50 Somogyi $u / d l$, a blood urea of $13.6 \mathrm{mmol} / 1$, haemoglobin of $10.9 \mathrm{~g} / \mathrm{l}$, and a white cell count of $14.7 \times 10^{6} / 1$. A plain abdominal radiograph showed a ground glass appearance, with multiple small fluid levels, and absence of the left psoas shadow (Fig. 1). $\stackrel{\overrightarrow{\vec{D}}}{\vec{\omega}}$ These findings suggested a strangulated left inguinal ${ }^{\omega}$ hernia. After resuscitation with intravenous fluids the patient was taken to theatre.

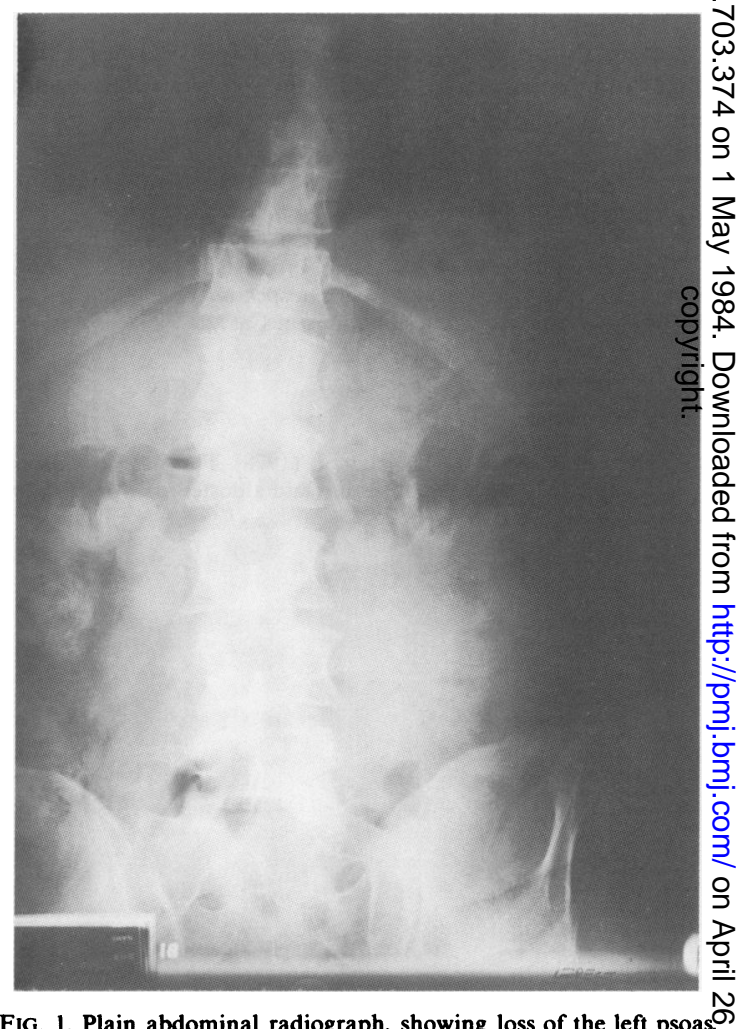

FIG. 1. Plain abdominal radiograph, showing loss of the left psoas shadow.

The left groin was explored via a supra-inguinal incision. The fascial layers of the spermatic cord ando the tunica vaginalis were necrotic but the testis and $\mathbb{\Phi}$ vessels of the spermatic cord were normal. There was:no hernia but considerable local oedema, and it was? 
evident that the problem originated within the abdomen. A lower midline incision was made and the abdomen explored. There was a considerable amount of blood stained ascitic fluid. The pancreas had undergone almost complete necrosis. It was evident that the cause of the left inguinal pathology was blood and pancreatic juices tracking retroperitoneally down the left paravertebral gutter, along the spermatic cord, and into the scrotum. The gall bladder was normal.

The abdomen was closed after peritoneal lavage and the insertion of drains.

Despite postoperative intermittent positive pressure ventilation and intensive care which included both haemodialysis and total parenteral nutrition, the patient died from multiple organ failure 16 days postoperatively. A post-mortem confirmed the diagnosis of acute haemorrhagic pancreatitis. A subsequent history from his family revealed that his alcohol intake was four bottles of spirits per week.

\section{Discussion}

Pancreatitis was first accurately described by Nikolaus Friedreich, Virchow's successor as Professor of Pathological Anatomy, University of Wurzburg, in 1878. Since then two well-known abdominal signs associated with the diagnosis of the disease have been described, the best known of these some 30 years after Friedreich. In 1920 George Grey Turner, a Newcastle upon Tyne and London surgeon, described local discoloration of the abdominal wall in two cases of acute pancreatitis (Grey Turner, 1919-20). In one patient there was discoloration around the umbilicus, and in the other, discoloration in the loins. He suggested that these were useful signs of the disease, and were due to the pancreatic ferments tracking through the layers of the abdominal wall. He felt, as a consequence of this, that they were probably related to the severity of the inflammation. Thomas Stephen Cullen, a Baltimore gynaecologist, had described periumbilical disco- loration in a case of ruptured ectopic pregnancy a year earlier (Cullen, 1919).

Blood, oedema and ascitic fluid, are well known for tracking into the scrotum but we have found only one previous report in the literature when pancreatitis presented as a swelling in the scrotum. This was a description by Zinin, Satsukevitch and Mokhanov (1979) of a patient in the U.S.S.R. who developed pancreatitis whilst in a psychiatric hospital. Similar to our patient, necrosis of the tunica vaginalis with a normal testis was found at operation.

The incidence of pancreatitis does not seem to be falling despite the present aggressive surgical policy towards the treatment of gall stones, and there is good evidence to suggest that in the areas of the country where alcohol is a major cause, that it is on the increase (Trapnell and Duncan, 1975; Imrie, 1975). Our case emphasises the well-known diagnostic pitfalls and the need to recognize another abdominal sign indicating severe disease.

\section{Acknowledgments}

We would like to thank Mr N. E. Dudley for permission to present this patient.

\section{References}

CUllen, T.S. (1919) Bluish discoloration of the umbilicus as a diagnostic sign where ruptured extra-uterine pregnancy exists. Contributions to Medical and Biological Research. Sir W. Osler, N.Y., i, 420.

IMRIE, C.W. (1975) Observations on acute pancreatitis. British Journal of Surgery, 61, 539.

Joshi, R.A., Probstein, J.G. \& Blumenthal, H.T. (1957) A survey of experience with three hundred clinical and eighty autopsy cases of acute pancreatitis. American Surgery, 23, 34.

Trapnell, J.E. \& DunCan, E.H.L. (1975) Patterns of incidence in acute pancreatitis. British Medical Journal, 2, 179.

TURNER, G. GREY (1919-20) Local discoloration of the abdominal wall as a sign of acute pancreatitis. British Journal of Surgery, vii, 394.

ZININ, A.F., SATSUKEVITCH, V.N. \& MoKhaNOR, N.P. (1979) Acute pancreatitis with haemorrhagic flow into the scrotum. Vestnik Khirurgiimeni, I. I. Grekova. 122, 47.

(Accepted 15 June 1983) 